\title{
Rejection of Limit Cycles Induced From Disturbance Observers in Motion Control
}

\author{
Rong-Hwang Horng, Student Member, IEEE, Heng-Lung Chou, and An-Chen Lee, Member, IEEE
}

\begin{abstract}
A disturbance observer (DOB) is generally introduced into motion control systems to eliminate the unwanted disturbance and plant uncertainty. Due to the quantization error from the sensors and D/A converter, DOB-based controller might generate limit cycles which severely decreases positioning accuracy as the system reaches steady-state positioning. This paper presents a sufficient condition for removing limit cycles and explores the condition to design controller parameters. In addition, since the parameters based on the condition make the system sluggish, an adaptive mechanism is introduced not only to maintain the system performance but also to eliminate limit cycles. The experimental results validate the analytical results and also illustrate the effectiveness of the proposed method.
\end{abstract}

Index Terms-Disturbance observer (DOB), limit cycle, linear-motor-driven motion system.

\section{INTRODUCTION}

$\mathbf{I}$ $\mathrm{N}$ RECENT years, a disturbance observer (DOB) is generally introduced into motion control systems to eliminate the "equivalent disturbance" as much as possible, and to force the actual system to become a nominal model. The equivalent disturbance consists of external-disturbance signals including friction, and signals due to model uncertainties and nonlinearity. If the uncertainties are removed by the DOB, the linear feedback controller can be applied to construct an asymptotically stable system. This equivalent disturbance was first introduced by Ohnishi [1] and refined by Umeno and Hori [2]. For improving performance in tracking and pointto-point control, Lee and Tomizuka [3] and other researchers like Komada et al. [4], White et al. [5], Shahruz [6], and Iwasaki et al. [7] demonstrated the effectiveness of the DOB by experiments with various uncertainties and external disturbances. However, the DOB has a drawback in steady-state positioning. A limit-cycle oscillation might occur while the system approaches the setpoint. Kobayashi et al. [8] judged that this oscillation is caused by the pole close to $z=-1$ that is introduced by the plant inversion in the DOB and encoder quantization error. However, no analytical results are provided concerning the mutual effect between the DOB and the quantization from the sensors and D/A converter. The authors also intuitively claimed that this problem can be solved by moving

Manuscript received September 25, 2004; revised April 25, 2005. Abstract published on the Internet September 15, 2006. This work was supported by the National Science Council of the Republic of China under Contract NSC922212-E009-018.

The authors are with the Department of Mechanical Engineering, National Chiao-Tung University, Hsinchu 300, Taiwan, R.O.C. (e-mail: aclee@mail.nctu.edu.tw)

Digital Object Identifier 10.1109/TIE.2006.885119 the DOB pole near $z=-1$ toward the inside of the unit circle by modification of the nominal plant model. Their experimental results show that the limit cycle can be suppressed without any significant changes in positioning accuracy by this approach. On the other hand, Fischer and Tomizuka [9] investigate highspeed positioning systems containing a DOB-based controller in an $x-y$ table driven over a ball-screw-nut mechanism by a dc servo motor along with two sensor systems, i.e., digital encoder and laser interferometer. They reported that the compliance of the coupling and the backlash increase the plant-model uncertainty and may cause limit cycles. A series of experiments was conducted, and consequently, the optimal controller settings in tracking and point to point were determined for the alternative position sensors. As stated above, limit cycles may be induced by various factors in motion control systems. The focus of this paper is to investigate the effect of quantization from the sensors and D/A converter on the occurrence of limit cycles in a positioning system. The experiments were conducted in a linear-motor-driven system for excluding nonlinear effects induced by the existing backlash and flexibility in a traditional ball-screw-driven system. The theorem in [10] is employed to derive sufficient conditions to avoid the limit cycles induced by the DOB and quantization. This sufficient condition will help us find adequate controller parameters in velocity loop for taking out limit cycles in nonlinear discrete time systems. After that, we will introduce an adaptive parameter tuning mechanism to maintain the tracking performance in transient state, and eliminate the limit cycle in steady state. Simulation and experiments are illustrated to demonstrate the effectiveness of the proposed method in the end.

\section{Sufficient Condition for Absence OF LIMIT CYCLE}

The controller structure adopted in our motion system is illustrated in Fig. 1. The controller of $C_{\mathrm{p}}(z)$ and $C_{\nu}(z)$ in position loop and velocity loop, respectively, can be any common used controller except integrator in position loop, which will induce severe limit cycles caused by friction [11]. Therefore, $\mathrm{P}$ control is the recommended position loop controller. The velocity loop controller is PI control. The feed-forward controller $F_{\mathrm{p}}(z)$ in position loop is a proper realization of a pure differentiator, while $F_{\nu}(z)$ in velocity loop is a proper realization of the inverse of the nonminimum phase plant $P(s)$. The friction compensator (FC) based on dynamic friction models, the LueGre model, is a feedforward type [12]. A position command illustrated in Fig. 2 is given to the system, and its corresponding velocity command is shown in Fig. 3. In our 


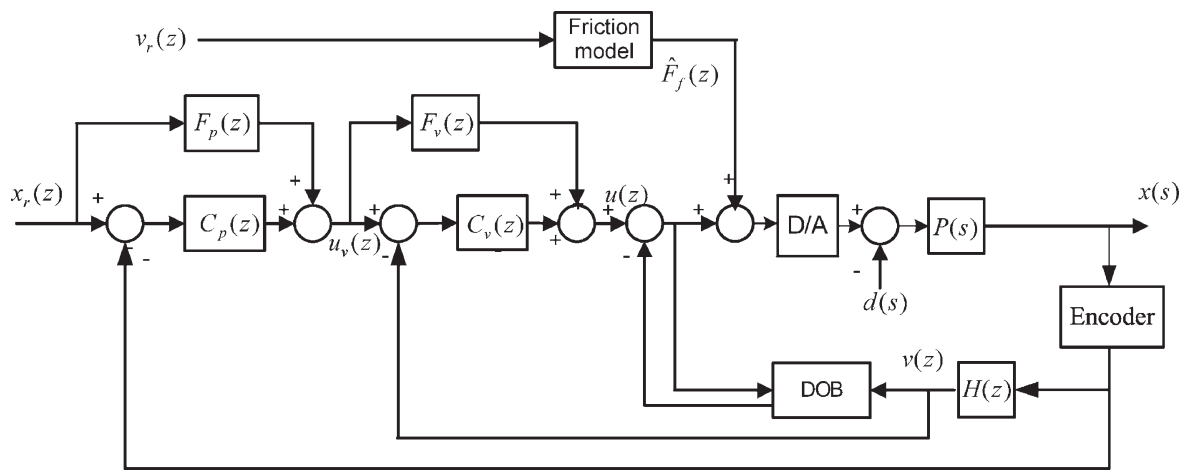

Fig. 1. Controller structure and plant of the experiment.

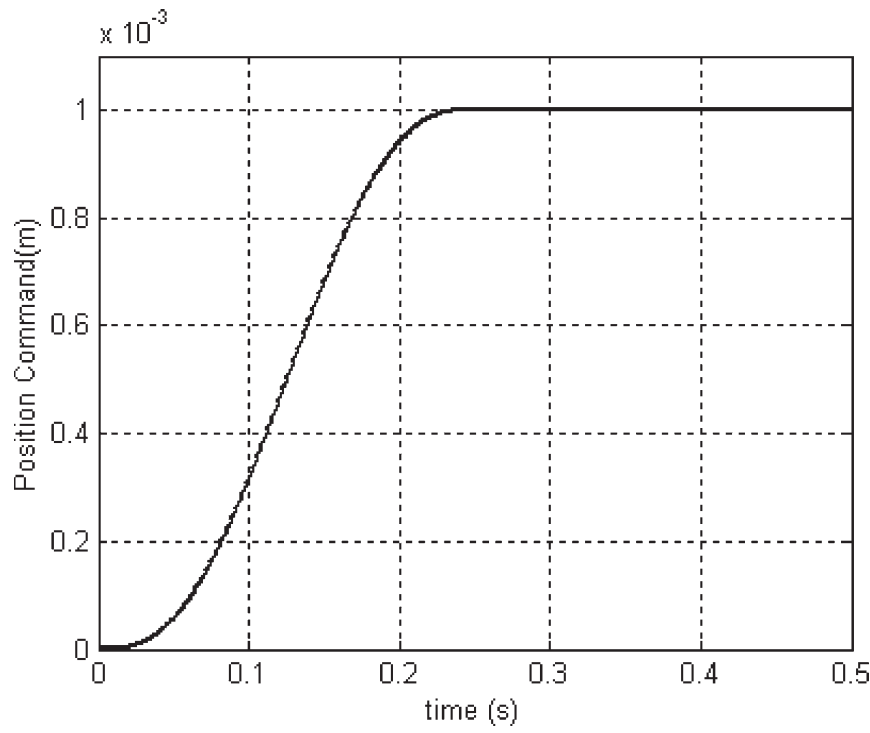

Fig. 2. Typical position command in positioning control.

motion system as shown in Fig. 1, when the system approaches the setpoint after acceleration-deceleration mechanism within $0-0.25 \mathrm{~s}$, the velocity command $u_{\nu}(z)$ fed into the velocity loop is almost zero because the velocity command coming from the feed-forward controller $F_{\mathrm{p}}(z)$ is zero, and the signal from $C_{\mathrm{p}}(z)$ is extremely small. At this moment, the signal-tonoise ratio in the velocity loop is very low that the precision of the system is seriously affected by the quantization error coming from encoder and digital-to-analog converter (DAC). Under the circumstances, the velocity loop, which has a DOB inside, is sustained to correct the errors during the steady-state positioning and might generate anxiety limit cycles if improper controller parameters are employed.

Therefore, for derivation of sufficient conditions to avoid the limit cycle induced by the DOB and quantization, we will focus our attention to the velocity loop that has DOB in the inner loop as shown in Fig. 4, and utilize the theorem in [10] for removing zero-input limit cycles in nonlinear discrete time systems. Here, we have two assumptions in our analysis.

1) External-disturbance signals including friction can be compensated completely by feed-forward-type compensator or DOB.

2) The limit cycle caused by the mutual effect between the DOB and quantization only has harmonic components

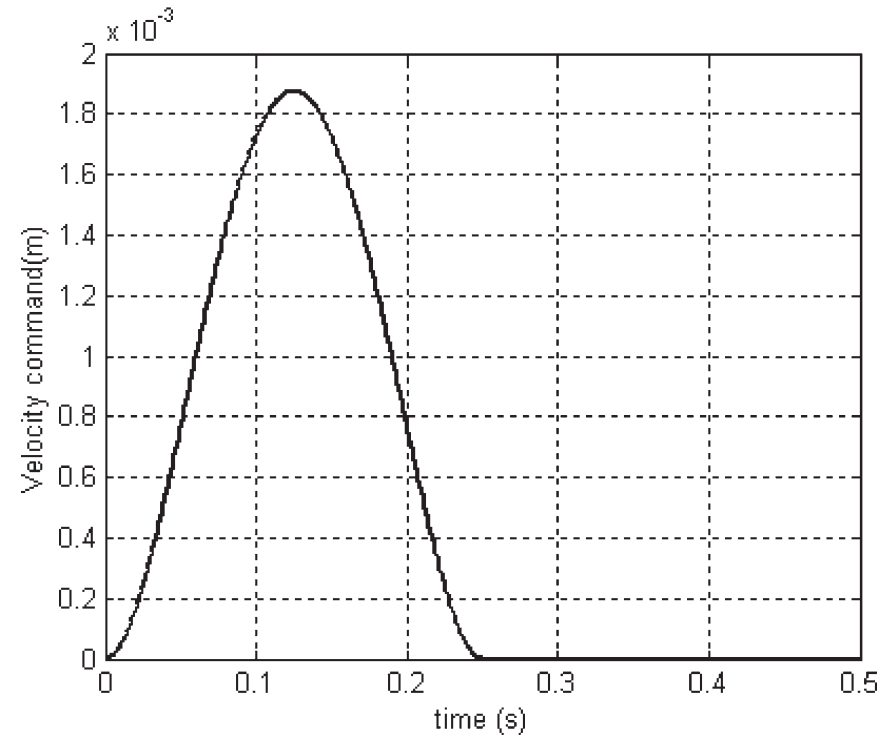

Fig. 3. Velocity command corresponds to position command in Fig. 2.

with no dc component, i.e., a pure periodic unbiased signal. This assumption makes sense when the experimental results in Section $\mathrm{V}$ are presented.

In Fig. 4, $H(z)$ denotes the discrete transfer function from feedback-sensor output to velocity $V(z)$. If the feedback sensor is a tachometer, $H(z)$ would be a constant. When a motor encoder is used as in most cases, $H(z)$ is some type of filter to estimate velocity. $Q_{1}$ and $Q_{2}$ are truncation quantizers from sensor and from DAC, respectively. The quantizers $Q_{1}$ and $Q_{2}$ conform to both of the two conditions listed as

$$
\begin{aligned}
Q(0) & =0 \\
0 & \leq \frac{Q(x)}{x} \leq 1 \quad \forall x \neq 0 .
\end{aligned}
$$

If the system response exhibits a limit cycle of which period length is $N$ samples, a function $\Lambda$ is defined as

$$
\begin{aligned}
\Lambda=N \cdot \sum_{k=0}^{N-1}\left\{Q_{1}[\right. & \left.x_{1}(k)\right] \cdot\left(x_{1}(k)-Q_{1}\left[x_{1}(k)\right]\right) \\
& \left.+Q_{2}\left[x_{2}(k)\right] \cdot\left(x_{2}(k)-Q_{2}\left[x_{2}(k)\right]\right)\right\}
\end{aligned}
$$




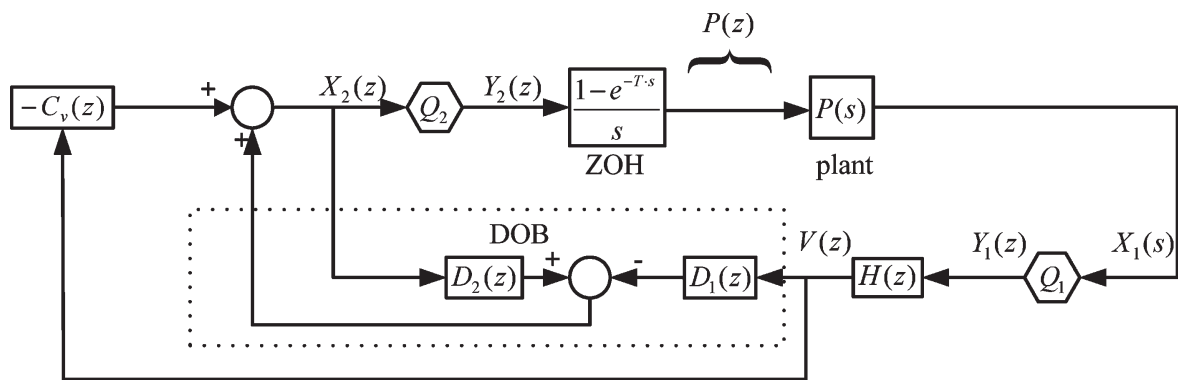

Fig. 4. Velocity loop of the controller structure in the Fig. 1.

where $x_{1}(k)$ is the signal before $Q_{1}$ in time domain and $x_{2}(k)$ denotes the signal before $Q_{2}$ in time domain. According to (1) and (2), the function $\Lambda$ is always semipositive and can be rewritten as

$$
\begin{aligned}
\Lambda=N \cdot \sum_{k=0}^{N-1}\left\{y_{1}(k) \cdot\left(x_{1}(k)-y_{1}(k)\right)\right. & \\
& \left.+y_{2}(k) \cdot\left(x_{2}(k)-y_{2}(k)\right)\right\} \geq 0
\end{aligned}
$$

where $y_{1}(k)$ denotes the signal after $Q_{1}$ in time domain and $y_{2}(k)$ indicates the signal after $Q_{2}$ in time domain. Then, discrete Parseval's theorem is utilized to transform the function $\Lambda$ into frequency-domain representation

$$
\begin{aligned}
\Lambda=\sum_{l=0}^{N-1}\left\{Y_{1}^{*}\left(z_{l}\right) \cdot\left(X_{1}\left(z_{l}\right)-Y_{1}\left(z_{l}\right)\right)\right. \\
\left.\quad+Y_{2}^{*}\left(z_{l}\right) \cdot\left(X_{2}\left(z_{l}\right)-Y_{2}\left(z_{l}\right)\right)\right\} \geq 0 \\
=\sum_{l=0}^{N-1}\left\{Y_{1}^{*}\left(z_{l}\right) \cdot\left(P\left(z_{l}\right) \cdot Y_{2}\left(z_{l}\right)-Y_{1}\left(z_{l}\right)\right)\right. \\
\left.\quad+Y_{2}^{*}\left(z_{l}\right) \cdot\left(B\left(z_{l}\right) \cdot Y_{1}\left(z_{l}\right)-Y_{2}\left(z_{l}\right)\right)\right\} \geq 0
\end{aligned}
$$

where $z_{l}=e^{j \cdot(2 \cdot \pi / N) \cdot l}, B\left(z_{l}\right)$ is the discrete transfer function from $Y_{1}\left(z_{l}\right)$ to $X_{2}\left(z_{l}\right)$. Equation (5) can be written by the matrix form

$\Lambda=\sum_{l=0}^{N-1}\left[Y_{1}^{*}\left(z_{l}\right) \quad Y_{2}^{*}\left(z_{l}\right)\right] \cdot\left[\begin{array}{cc}-1 & P\left(z_{l}\right) \\ B\left(z_{l}\right) & -1\end{array}\right] \cdot\left[\begin{array}{c}Y_{1}\left(z_{l}\right) \\ Y_{2}\left(z_{l}\right)\end{array}\right] \geq 0$.

Let $\vec{Y}\left(z_{l}\right)=\left[\begin{array}{ll}Y_{1}\left(z_{l}\right) & Y_{2}\left(z_{l}\right)\end{array}\right]^{\mathrm{T}}, \vec{Y}^{*}\left(z_{l}\right)$ denotes the conjugate complex transpose of $\vec{Y}\left(z_{l}\right)$, and $A\left(z_{l}\right)=\left[\begin{array}{cc}-1 & P\left(z_{l}\right) \\ B\left(z_{l}\right) & -1\end{array}\right]$. Equation (6) can be rewritten as

$$
\Lambda=\sum_{l=0}^{N-1} \vec{Y}^{*}\left(z_{l}\right) \cdot A\left(z_{l}\right) \cdot \vec{Y}\left(z_{l}\right) .
$$

Furthermore, (7) can be arranged in the form

$\Lambda=\sum_{l=0}^{N-1}\left[\vec{Y}^{*}\left(z_{l}\right) \cdot A_{\mathrm{H}}\left(z_{l}\right) \cdot \vec{Y}\left(z_{l}\right)+\vec{Y}^{*}\left(z_{l}\right) \cdot A_{\mathrm{SH}}\left(z_{l}\right) \cdot \vec{Y}\left(z_{l}\right)\right]$ where $A_{\mathrm{H}}\left(z_{l}\right)=(1 / 2) \cdot\left(A\left(z_{l}\right)+A^{*}\left(z_{l}\right)\right)$ is Hermitian part of $A\left(z_{l}\right)$, and $A_{\mathrm{SH}}\left(z_{l}\right)=(1 / 2) \cdot\left(A\left(z_{l}\right)-A^{*}\left(z_{l}\right)\right)$ is skew Hermitian part of $A\left(z_{l}\right)$.

Because the value of $\vec{Y}^{*}\left(z_{l}\right) \cdot A_{\mathrm{H}}\left(z_{l}\right) \cdot \vec{Y}\left(z_{l}\right)$ is only real and $\vec{Y}^{*}\left(z_{l}\right) \cdot A_{\mathrm{SH}}\left(z_{l}\right) \cdot \vec{Y}\left(z_{l}\right)$ is zero or pure imaginary, $\vec{Y}^{*}\left(z_{l}\right)$. $A_{\mathrm{H}}\left(z_{l}\right) \cdot \vec{Y}\left(z_{l}\right)$ is the real part of $\vec{Y}^{*}\left(z_{l}\right) \cdot A\left(z_{l}\right) \cdot \vec{Y}\left(z_{l}\right)$, and $\vec{Y}^{*}\left(z_{l}\right) \cdot A_{\mathrm{SH}}\left(z_{l}\right) \cdot \vec{Y}\left(z_{l}\right)$ is the imaginary part of $\vec{Y}^{*}\left(z_{l}\right)$. $A\left(z_{l}\right) \cdot \vec{Y}\left(z_{l}\right)$, i.e.

$$
\begin{aligned}
\vec{Y}^{*}\left(z_{l}\right) \cdot A_{\mathrm{H}}\left(z_{l}\right) \cdot \vec{Y}\left(z_{l}\right) & =\operatorname{Re}\left(\vec{Y}^{*}\left(z_{l}\right) \cdot A\left(z_{l}\right) \cdot \vec{Y}\left(z_{l}\right)\right) \\
\vec{Y}^{*}\left(z_{l}\right) \cdot A_{\mathrm{SH}}\left(z_{l}\right) \cdot \vec{Y}\left(z_{l}\right) & =\operatorname{Im}\left(\vec{Y}^{*}\left(z_{l}\right) \cdot A\left(z_{l}\right) \cdot \vec{Y}\left(z_{l}\right)\right) .
\end{aligned}
$$

In (7), the values of $\vec{Y}^{*}\left(z_{l}\right) \cdot A\left(z_{l}\right) \cdot \vec{Y}\left(z_{l}\right)$ for $l=1 \sim((N / 2)-1) \quad$ and $\quad l=((N / 2)+1) \sim(N-1)$ are conjugate complex. Therefore, function $\Lambda$ can be rewritten by the form, or $\Lambda=\vec{Y}^{*}\left(z_{0}\right) \cdot A\left(z_{0}\right) \cdot \vec{Y}\left(z_{0}\right)+2 \cdot \sum_{l=1}^{(N / 2)-1}$ $\operatorname{Re}\left(\vec{Y}^{*}\left(z_{l}\right) \cdot A\left(z_{l}\right) \cdot \vec{Y}\left(z_{l}\right)\right)+\vec{Y}^{*}\left(z_{N / 2}\right) \cdot A\left(z_{N / 2}\right) \cdot \vec{Y}\left(z_{N / 2}\right)$. Since the limit cycle is assumed to be a pure harmonic signal, i.e., $\vec{Y}\left(z_{0}\right)=\overrightarrow{0}$, and $\vec{Y}^{*}\left(z_{N / 2}\right) \cdot A\left(z_{N / 2}\right) \cdot \vec{Y}\left(z_{N / 2}\right)$ only has real part, the above equation can be rewritten as

$$
\begin{aligned}
\Lambda=2 \cdot \sum_{l=1}^{\frac{N}{2}-1} \operatorname{Re} & \left(\vec{Y}^{*}\left(z_{l}\right) \cdot A\left(z_{l}\right) \cdot \vec{Y}\left(z_{l}\right)\right) \\
& +\operatorname{Re}\left(\vec{Y}^{*}\left(z_{N / 2}\right) \cdot A\left(z_{N / 2}\right) \cdot \vec{Y}\left(z_{N / 2}\right)\right) .
\end{aligned}
$$

If we substitute (9) into (11), we find that

$$
\begin{aligned}
\Lambda=2 \cdot \sum_{l=1}^{\frac{N}{2}-1} \vec{Y}^{*}\left(z_{l}\right) & \cdot A_{\mathrm{H}}\left(z_{l}\right) \cdot \vec{Y}\left(z_{l}\right) \\
& +\vec{Y}^{*}\left(z_{N / 2}\right) \cdot A_{\mathrm{H}}\left(z_{N / 2}\right) \cdot \vec{Y}\left(z_{N / 2}\right) .
\end{aligned}
$$

Given that $A_{\mathrm{H}}\left(z_{l}\right)$ is negative definite (i.e., $A_{\mathrm{H}}\left(z_{l}\right)<0$ ) for $l=1 \sim(N / 2)$, then the right-hand side of (12) is always seminegative, while the left-hand side $\Lambda$ is always semipositive. The only condition to satisfy both sides is $\vec{Y}\left(z_{l}\right)=\overrightarrow{0}$ for $l=$ $1 \sim(N / 2)$, and consequently, no limit cycles will generate. Because negative definite of $A_{\mathrm{H}}\left(z_{l}\right)$ is the sufficient condition for eliminating zero-input limit cycles, we could design the 


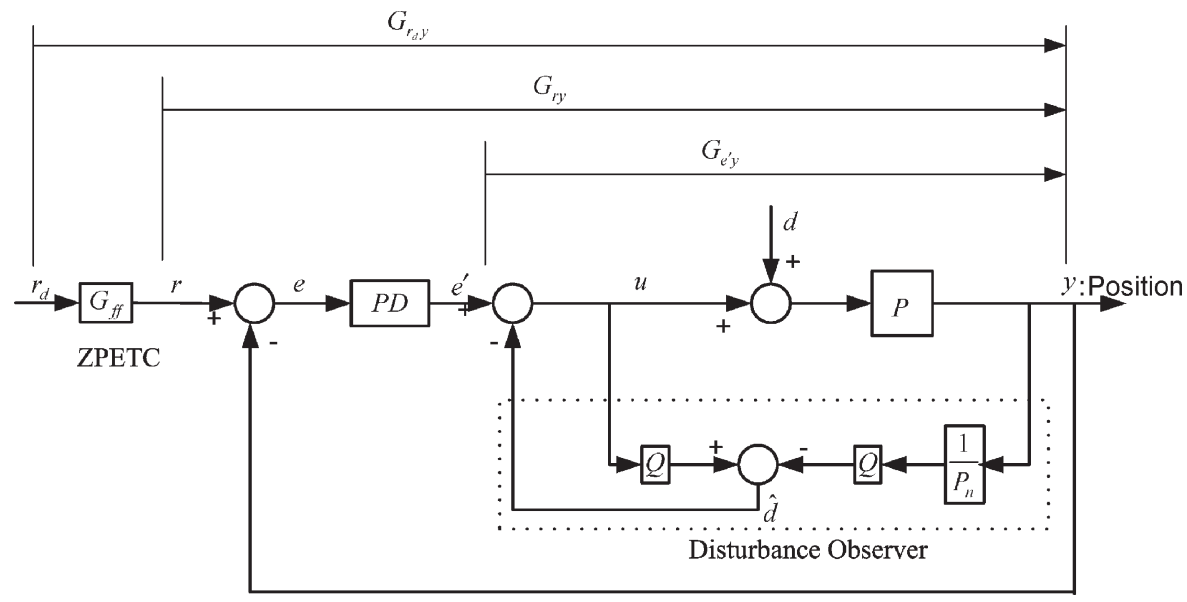

Fig. 5. Motion control structure proposed by Kobayashi [8].
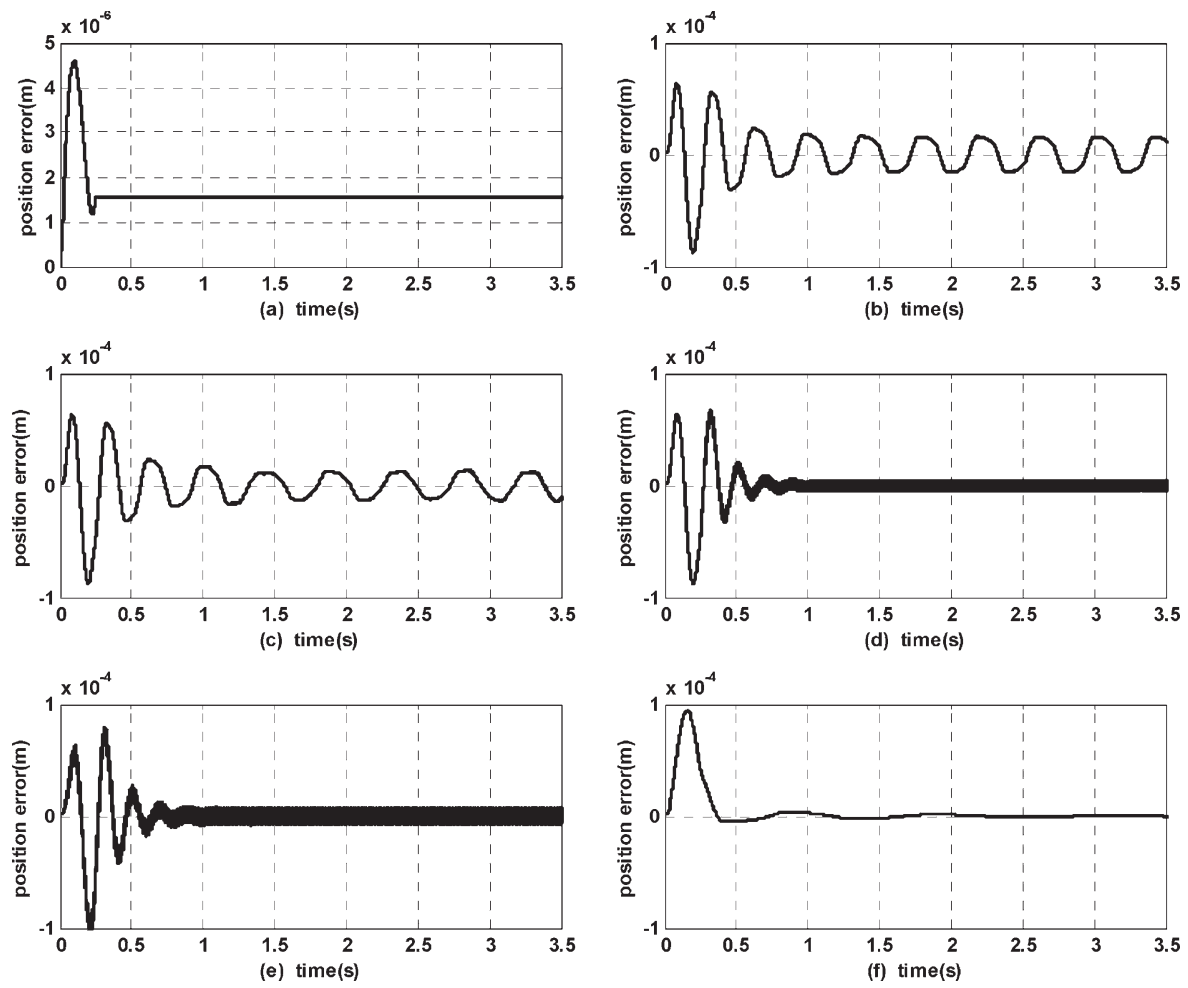

Fig. 6. Simulation results for the six controller structures. (a) PD. (b) PD + DOB (pole near -1 ). (c) PD + DOB (pole at -0.79 ). (d) PD + DOB (pole at -0.6 ). (e) PD + DOB (pole at -0.4). (f) PD + DOB (pole at 0.98).

controller parameters to make $A_{\mathrm{H}}\left(z_{l}\right)$ negative definite for $l=$ $1 \sim(N / 2)$, in order to avoid limit cycles.

The eigenvalue of $A_{\mathrm{H}}\left(z_{l}\right)$ can be obtained as follows:

$$
\begin{aligned}
& A_{\mathrm{H}}\left(z_{l}\right)=\left[\begin{array}{cc}
-1 & \frac{P\left(z_{l}\right)+B^{*}\left(z_{l}\right)}{2} \\
\frac{\left(P\left(z_{l}\right)+B^{*}\left(z_{l}\right)\right)^{*}}{2} & -1
\end{array}\right] \\
& \left|\lambda \cdot I-A_{\mathrm{H}}\left(z_{l}\right)\right|=0 \\
& \left|\begin{array}{cc}
\lambda+1 & -\frac{\left(P\left(z_{l}\right)+B^{*}\left(z_{l}\right)\right)}{2} \\
-\frac{\left(P\left(z_{l}\right)+B^{*}\left(z_{l}\right)\right)^{*}}{2} & +1
\end{array}\right|=0 \\
& \lambda^{2}+2 \cdot \lambda+1-\frac{\left|P\left(z_{l}\right)+B^{*}\left(z_{l}\right)\right|^{2}}{4}=0 .
\end{aligned}
$$

If and only if all coefficients in the polynomial (13) are positive, the eigenvalue $\lambda$ of $A_{\mathrm{H}}\left(z_{l}\right)$ is negative. This implies that if the following conditions are satisfied, or

$$
\begin{aligned}
1-\frac{\left|P\left(z_{l}\right)+B^{*}\left(z_{l}\right)\right|^{2}}{4} & >0 \\
\frac{\left|P\left(z_{l}\right)+B^{*}\left(z_{l}\right)\right|^{2}}{4} & <1 \\
\left|P\left(z_{l}\right)+B^{*}\left(z_{l}\right)\right| & <2
\end{aligned}
$$

then $A_{\mathrm{H}}\left(z_{l}\right)$ is negative definite Hermitian matrix and satisfies the sufficient condition for removing limit cycles. Now, we will 
obtain the discrete transfer function $B\left(z_{l}\right)$. Let us take a close look at the path from $Y_{1}\left(z_{l}\right)$ to $X_{2}\left(z_{l}\right)$ in Fig. 4 as follows:

$$
\begin{aligned}
-Y_{1}\left(z_{l}\right) \cdot H\left(z_{l}\right) \cdot C_{\nu}\left(z_{l}\right) & \\
+ & {\left[X_{2}\left(z_{l}\right) \cdot D_{2}\left(z_{l}\right)-Y_{1}\left(z_{l}\right) \cdot H\left(z_{l}\right) \cdot D_{1}\left(z_{l}\right)\right] } \\
& =X_{2}\left(z_{l}\right) \\
X_{2}\left(z_{l}\right) & =\left[\frac{H\left(z_{l}\right) \cdot C_{\nu}\left(z_{l}\right)+H\left(z_{l}\right) \cdot D_{1}\left(z_{l}\right)}{D_{2}\left(z_{l}\right)-1}\right] \cdot Y_{1}\left(z_{l}\right)
\end{aligned}
$$

so

$$
B\left(z_{l}\right)=\left[\frac{H\left(z_{l}\right) \cdot C_{\nu}\left(z_{l}\right)+H\left(z_{l}\right) \cdot D_{1}\left(z_{l}\right)}{D_{2}\left(z_{l}\right)-1}\right] .
$$

Substituting the discrete transfer function $B\left(z_{l}\right)$ of (15) into (14) yields

$$
\left|P\left(z_{l}\right)+\left(\frac{H\left(z_{l}\right) \cdot C_{\nu}\left(z_{l}\right)+H\left(z_{l}\right) \cdot D_{1}\left(z_{l}\right)}{D_{2}\left(z_{l}\right)-1}\right)^{*}\right|<2
$$

for $l=1 \sim(N / 2)\left(z_{l}=e^{j \cdot(2 \cdot \pi / N) \cdot l}\right)$.

Theorem: As demonstrated in Fig. 4, the $N$ length limit cycles induced by DOB and quantization are absent from the velocity loop if the following condition is satisfied:

$$
\begin{aligned}
&\left|P\left(z_{l}\right)+\left(\frac{H\left(z_{l}\right) \cdot C_{\nu}\left(z_{l}\right)+H\left(z_{l}\right) \cdot D_{1}\left(z_{l}\right)}{D_{2}\left(z_{l}\right)-1}\right)^{*}\right|<2 \\
& \text { for } \quad l=1 \sim \frac{N}{2}\left(z_{l}=e^{j \cdot(2 \cdot \pi / N) \cdot l}\right)
\end{aligned}
$$

(proof as above paragraph).

\section{PARAmeter Tuning For Motion CONTROL}

The general mathematical model of a motion system from driving voltage to motion position can be expressed as

$$
P(s)=\frac{k_{\mathrm{t}} \cdot k_{\mathrm{a}}}{s(J \cdot s+B)}
$$

where $J$ is the linear inertia $(\mathrm{Kg}), B$ is the viscous friction coefficient $(\mathrm{Kg} / \mathrm{s}), k_{\mathrm{a}}$ is the current driver gain $(\mathrm{A} / \mathrm{V})$, and $k_{\mathrm{t}}$ is the motor force constant (Nt/A). For verifying the validity of the inequality (16), three tuning algorithms to treat limit cycles are described in this section.

The first tuning algorithm is presented by Fischer and Tomizuka [9] concerning the feedback controller, DOB, and the type of position sensors. The overall controller structure under study is difficult to analyze because the compliance of the coupling and the backlash of the mechanical properties are involved in the forward loop. Tracking and point-to-point performance can be improved by choosing suitable controller parameters based on a series of experiments. Guidelines for the controller tuning are provided depending on the type of the position sensors and the control task. Improper-controllerparameter settings may reduce or enlarge the limit-cycle oscillation when the system approaches setpoint.

As to the second tuning algorithm proposed by Kobayashi et al. [8], the motion control structure is illustrated in Fig. 5. The motion system only has position loop with no velocity loop, so the $C_{\nu}\left(z_{l}\right)$ in the inequality (16) is set to zero. Since the inverse plant transfer function $P^{-1}(s)$ in the system is from position to voltage, not from velocity to voltage, the $H\left(z_{l}\right)$ equals one and the continuous transfer function $P^{-1}(s)$ is listed as

$$
P^{-1}(s)=\frac{s \cdot(J \cdot s+B)}{k_{\mathrm{t}} \cdot k_{\mathrm{a}}} .
$$

A third-order low-pass filter $Q(s)$ is used to make $P^{-1}(s)$ become realizable

$$
\begin{aligned}
Q(s) & =\frac{3 \cdot \mathrm{tau} \cdot s+1}{(\mathrm{tau} \cdot s+1)^{3}} \\
\mathrm{tau} & =\frac{1}{2 \cdot \pi \cdot B_{\mathrm{w}}}
\end{aligned}
$$

where $B_{\mathrm{w}}$ is the cutoff frequency $(\mathrm{Hz})$ of the low-pass filter.

In Kobayashi's work, the authors transferred the continuous transfer function $P(s)$ into a discrete transfer function $P(z)$ by zero-order hold method and found that $P(z)$ had zero near -1 . Consequently, $P^{-1}(z)$ in the DOB had pole near -1 [has pole near $\pm j\left(\omega_{s} / 2\right)$ in $\mathrm{S}$ domain and $\omega_{s}$ is its sampling frequency $(\mathrm{rad} / \mathrm{s})]$. They considered the outcome as the reason why the system generated limit cycles. Disregarding the nature of the system, they moved the pole of $P^{-1}(z)$ much more inside the unit circle by a parameter $r$ [see (17)].

$$
P^{-1}\left(z^{-1}\right)=\frac{1+a_{1} \cdot z^{-1}+a_{2} \cdot z^{-2}}{\left(b_{1}+r\right) \cdot z^{-1}+\left(b_{2}-r\right) \cdot z^{-2}} .
$$

Furthermore, the authors moved the pole of $P^{-1}(z)$ to -0.6 , and the experimental results showed that the limit cycles could be reduced (but not eliminated).

If we take $P^{-1}(z)$ with pole near -1 into the inequality (16), we are not able to find parameters which satisfy the sufficient condition, even if we lower the cutoff frequency of the low-pass filter in the DOB to a very low value (e.g., $5 \mathrm{~Hz}$ ). The solutions that can satisfy the sufficient condition are moving the pole of original $P^{-1}(z)$ to the right-hand side (approximately on the 0.98 in Z-plane), and $5 \mathrm{~Hz}$ of the lowpass filter cutoff frequency. Simulation with our motion system parameters is performed to compare Kobayashi's work with our method. Six controller structures are concerned, i.e., the position $\mathrm{PD}$ controller (PD), $\mathrm{PD}+\mathrm{DOB}$ with inverse plant pole near $-1, \mathrm{PD}+\mathrm{DOB}$ with modified inverse plant pole near $-0.78, \mathrm{PD}+\mathrm{DOB}$ with modified inverse plant pole near -0.6 , PD + DOB with modified inverse plant pole near -0.4 , and PD + DOB with modified inverse plant pole near 0.98 , driven by the S-curve position command.

$x_{r}(t)= \begin{cases}A\left[6\left(\frac{t}{T_{r}}\right)^{5}-15\left(\frac{t}{T_{r}}\right)^{4}+10\left(\frac{t}{T_{r}}\right)^{3}\right], & 0 \leq t \leq T_{\mathrm{acc}} \\ A, & t>T_{\mathrm{acc}}\end{cases}$

where $A=1 \times 10^{-3} \mathrm{~m}$ and $T_{\mathrm{acc}}=0.25 \mathrm{~s}$. Simulation results are shown in Fig. 6 with $40-\mathrm{Hz}$ PD bandwidth and 5-Hz lowpass filter cutoff frequency. If the pole of $P^{-1}(z)$ near $z=-1$ 


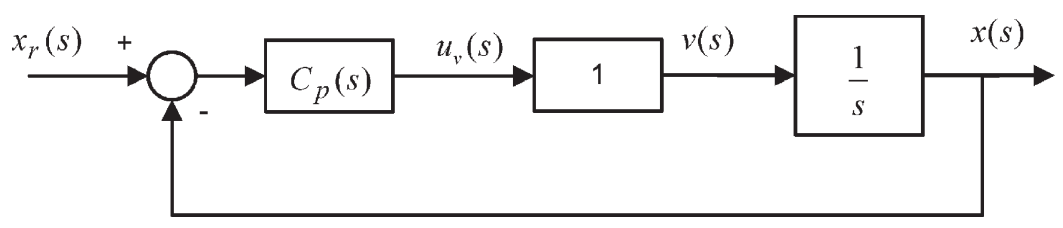

Fig. 7. Equivalent controller structure after acceleration-deceleration period.

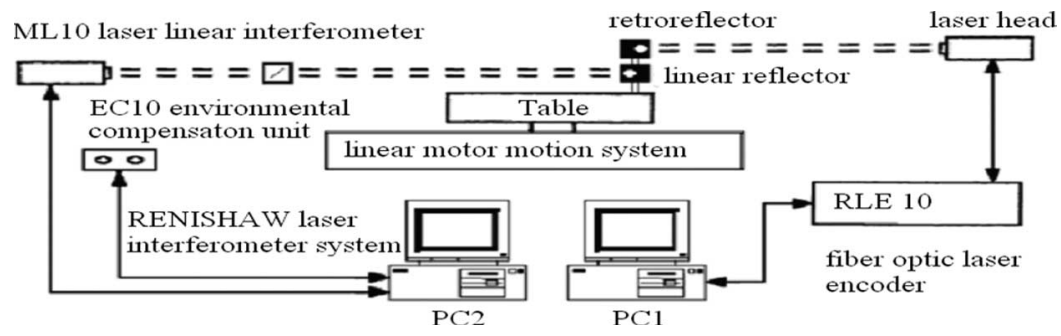

Fig. 8. Experimental linear-motor-driven motion system together with the resolution calibration system.

TABLE I

MOTION SYSTEM PARAMETERS

\begin{tabular}{|l|c|c|}
\hline \multicolumn{1}{|c|}{ Plant Parameters } & & Unit \\
\hline$J$, linear inertia & 2.49 & $\mathrm{Kg}$ \\
\hline$B$, viscous friction coefficient & 44.14 & $\mathrm{~kg} / \mathrm{sec}$ \\
\hline \hline$K_{t}$, force constant of motor & 28.5 & $\mathrm{~N} / \mathrm{A}$ \\
\hline$K_{a}$, gain of current driver & 0.349 & $\mathrm{~A} /$ Volt \\
\hline
\end{tabular}

TABLE II

ORIGINAL AND THE TUNED PARAMETERs FOR CASCADE CONTROL

\begin{tabular}{|l|c|c|c|}
\hline \multicolumn{1}{|c|}{ Parameters } & Original & Tuned & Unit \\
\hline$T$, sampling time & 0.0005 & 0.0005 & $\mathrm{sec}$ \\
\hline$J$, linear inertia & 2.49 & 2.49 & $\mathrm{Kg}$ \\
\hline$B$, viscous friction coefficient & 44.14 & 44.14 & $\mathrm{~kg} / \mathrm{sec}$ \\
\hline$K_{t}$, force constant of motor & 28.5 & 28.5 & $\mathrm{~N} / \mathrm{A}$ \\
\hline$K_{a}$, gain of current driver & 0.349 & 0.349 & $\mathrm{~A} / \mathrm{Volt}$ \\
\hline$k_{v p}$, P gain in the velocity loop & 184 & 0.025 & $\mathrm{Volt} \mathrm{sec} / \mathrm{m}$ \\
\hline$k_{v i}$, I gain in the velocity loop & 35537 & 1 & $\mathrm{Volt} / \mathrm{m}$ \\
\hline$B_{w}$, low-pass filter cut-off freq & 10 & 1 & $\mathrm{~Hz}$ \\
\hline$\beta$, parameter in the $\alpha-\beta$ filter & 0.32 & 0.001 & (none) \\
\hline
\end{tabular}

is moved away from -1 to -0.78 , the magnitude and frequency of limit-cycle oscillation is reduced but not eliminated. As the pole is moved further inside the unit circle, such as $z=-0.6$ or $z=-0.4$, high-frequency oscillation occurs during steadystate positioning. However, the limit-cycle oscillation can be eliminated if the pole $P^{-1}(z)$ near $z=-1$ is shifted to 0.98 to satisfy the sufficient condition. Therefore, the condition in (16) is much more stringent than that used by Kobayashi, but the ways to treat limit cycles happen to be the same on moving the pole to the right. Besides, the inverse plant $P^{-1}(z)$ used in our observer design (see Fig. 1) is transformed by polezero matched method and, the inverse plant does not have pole on the left-hand side like Kobayashi's systems. However, our system still generates limit cycles. In the next paragraph, we will present the way to design controller parameters (see Fig. 1) by inequality (16) for limit-cycles elimination.

As to our tuning algorithm, the velocity loop of the motion control structure can be shown in Fig. 4. The feedback sensor is encoder, so the discrete transfer function $H(z)$, i.e., $\alpha-\beta$ filter [13] is used to estimate the velocity $V(z)$. The $\alpha-\beta$ filter
TABLE III

TUNED PARAMETERS FOR LINEAR ADAPTIVE MECHANISM

\begin{tabular}{|l|c||c||c|}
\hline \multicolumn{1}{|c||}{ Parameters } & Original & Tuned & Unit \\
\hline$k_{v p}$, P gain in the velocity loop & 184 & 0.025 & Volt sec $/ \mathrm{m}$ \\
\hline$k_{v i}$, I gain in the velocity loop & 35537 & 1 & Volt $/ \mathrm{m}$ \\
\hline \hline$B_{w}$, low-pass filter cut-off freq & 10 & 1 & $\mathrm{~Hz}$ \\
\hline \hline$\beta$, parameter in the $\alpha-\beta$ filter & 0.32 & 0.001 & (none) \\
\hline$k_{p p}$, P gain in the position loop & 25 & 2000 & $1 / \mathrm{sec}$ \\
\hline
\end{tabular}

we used here is critical damping of a second-order type and is listed as

$$
H\left(z^{-1}\right)=\frac{\beta-\beta \cdot z^{-1}}{T+T \cdot(2 \cdot \sqrt{\beta}-2) \cdot z^{-1}+T \cdot(1-2 \cdot \sqrt{\beta}+\beta) \cdot z^{-2}}
$$

where $T$ indicates the sampling time and $\beta$ denotes a coefficient which can determine the cutoff frequency of the $\alpha-\beta$ filter.

$D_{1}(z)$ is the low-pass filter transfer function multiplied by the inverse plant transfer function (The inverse plant transfer function in the $D_{1}(z)$ is from velocity to voltage). We use polezero matched method to design $D_{1}(z)$ as

$$
\begin{aligned}
D_{1}\left(z^{-1}\right)= & \frac{B \cdot\left(1-e^{(-T / \mathrm{tau})}\right)^{2}}{k_{\mathrm{t}} \cdot k_{\mathrm{a}} \cdot\left(1-e^{(-B \cdot T / J)}\right)} \\
& \cdot \frac{\left(z^{-1}-e^{(-B \cdot T / J)} \cdot z^{-2}\right)}{\left(1-2 \cdot e^{(-T / \mathrm{tau})} \cdot z^{-1}+e^{(-2 \cdot T / \mathrm{tau})} \cdot z^{-2}\right)} \\
\operatorname{tau}= & \frac{1}{2 \cdot \pi \cdot B_{\mathrm{w}}}
\end{aligned}
$$

where $B_{\mathrm{w}}$ is low-pass filter cutoff frequency $(\mathrm{Hz})$.

$D_{2}(z)$ is the low-pass filter that is identical to the one in $D_{1}(z)$ and is shown as

$$
\begin{aligned}
D_{2}\left(z^{-1}\right)= & \frac{\left(1-e^{(-T / \mathrm{tau})}\right)^{2}}{2} \\
& \cdot \frac{\left(z^{-1}+z^{-2}\right)}{\left(1-2 \cdot e^{(-T / \mathrm{tau})} \cdot z^{-1}+e^{(-2 \cdot T / \mathrm{tau})} \cdot z^{-2}\right)}
\end{aligned}
$$




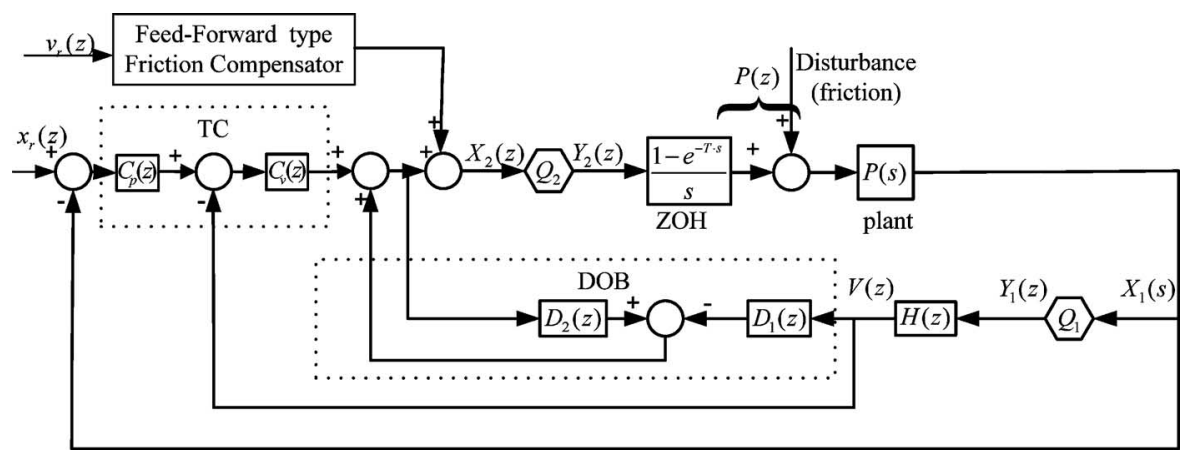

Fig. 9. Simulation structure of the motion system.
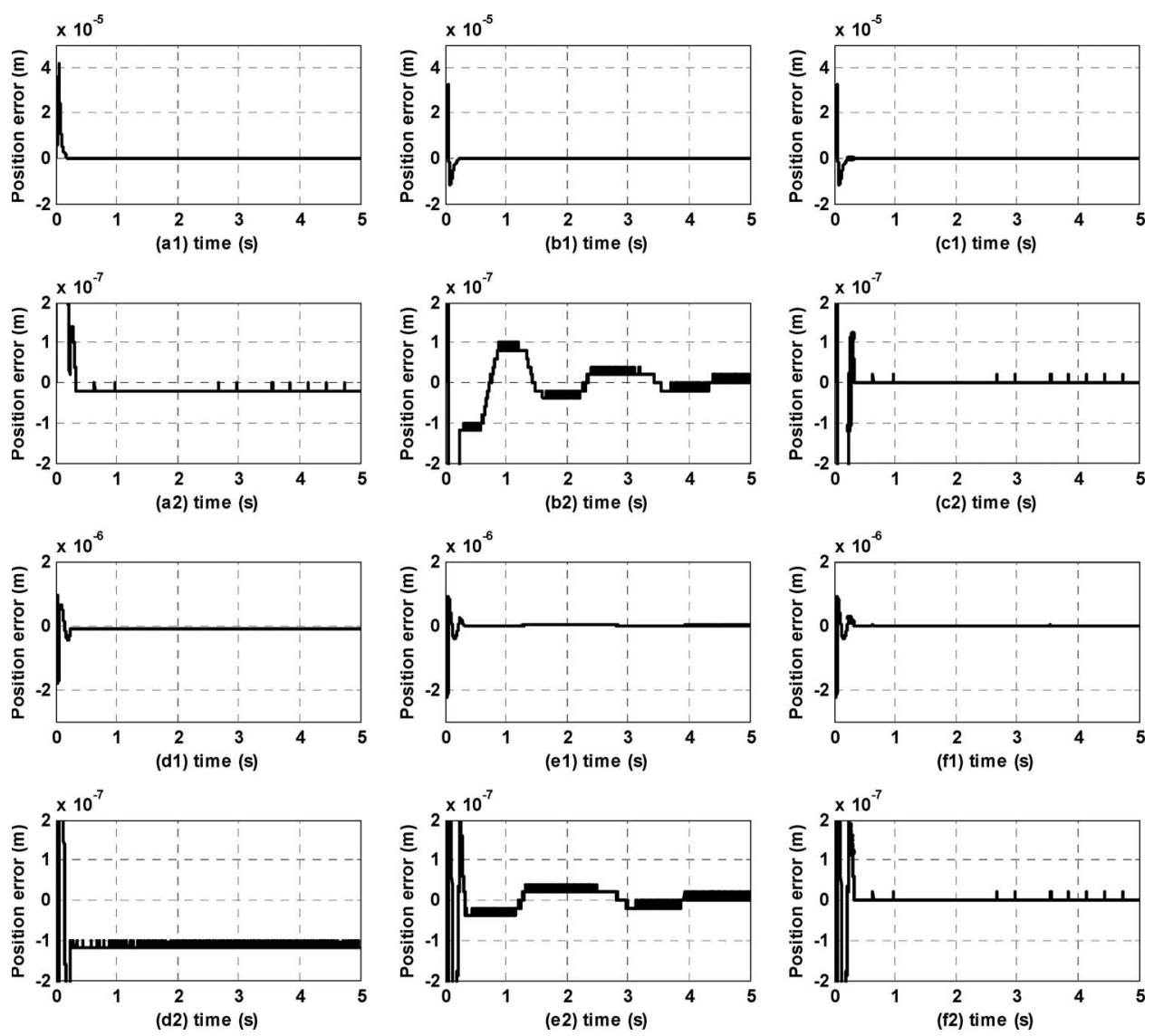

Fig. 10. Simulation results for the six controller structures. (a) TC. (b) TC + DOB with original parameters. (c) TC + DOB with adaptive mechanism. (d) TC + FC. (e) TC + FC + DOB with original parameters. (f) TC + FC + DOB with adaptive mechanism.

$C_{\nu}(z)$ in the velocity loop is PI control which is realized as follows:

$$
C_{\nu}\left(z^{-1}\right)=k_{\nu \mathrm{p}}+\frac{k_{\nu i} \cdot T}{1-z^{-1}}
$$

where $k_{\nu \mathrm{p}}$ and $k_{\nu i}$ are $\mathrm{P}$ gain and I gain in the velocity loop.

Now, we will obtain the plant transfer function $P(z)$ including zero-order hold (see Fig. 4). Take the z-transform of the signal $X_{1}(s)$ in (24), which is found at the bottom of the next page. Then, we can take the discrete transfer functions (19), (20), (22)-(24) into the inequality (16) to find the appropriate parameters.
Since the plant parameters, $J, B, k_{\mathrm{t}}$, and $k_{\mathrm{a}}$, are dependent on the characteristics of the system, we can only adjust the controlled parameters, $k_{\nu p}, k_{\nu i}, \beta$, and $B_{\mathrm{w}}$, for eliminating limit cycles. Let the limit cycles that occur in the system be a finite length (period) $N$. The larger the $N$ is, the more stringent the inequality (16) is. From our analytical results presented later in Section $\mathrm{V}$, we are aware that the value of the controlled parameters for eliminating the limit cycles is much smaller than the original one, and the system designed under these parameters has poor performance. Therefore, in the next section, we will propose an adaptive parameter tuning mechanism to tackle this problem. 


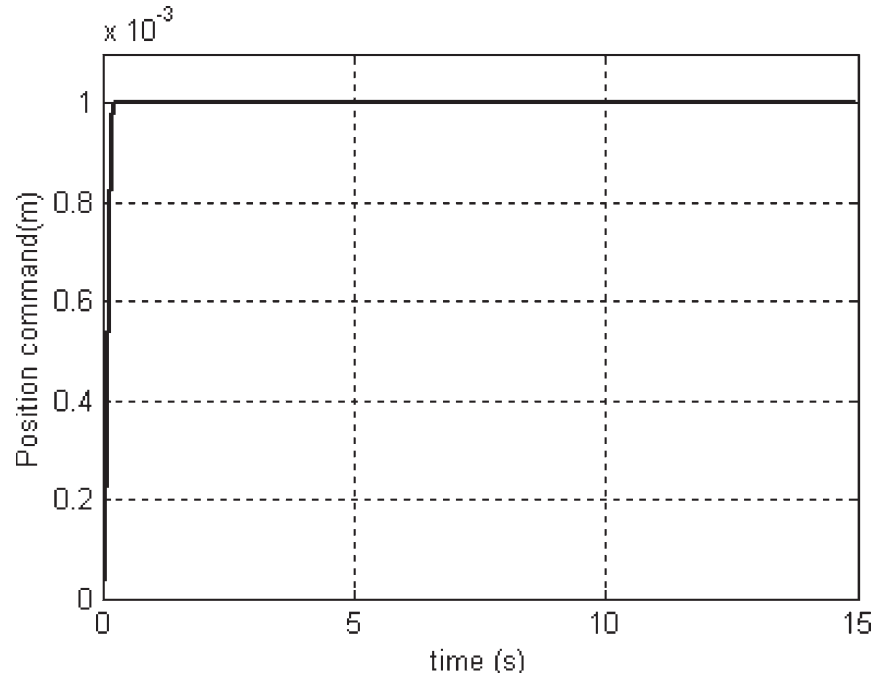

Fig. 11. Position command in Case (I).

\section{Adaptive Parameter Tuning Mechanism}

According to the parameters, we found by conforming to the inequality (16), the performance in the velocity loop is too poor to meet the performance requirement of the modern motion control system. The purpose of the adaptive parameter tuning mechanism is to maintain the performance of the system by keeping the original designed parameters during acceleration-deceleration period in the velocity loop. When the system gradually becomes steady state, we linearly tune the velocity loop parameters into the values for eliminating limit cycles without severely impacting the overall performance of the system, and linearly increase the $\mathrm{P}$ gain in the position loop to make the system rapidly approach the setpoint. The reason for increasing the $\mathrm{P}$ gain in the position loop is explained as follow. After acceleration-deceleration mechanism, the system is almost in the steady state. The velocity of the system is very slow in this phase, so the DOB, making the system become nominal plant, will actually take effect, and in addition, the feed-forward controller $F_{\nu}(s)$, which is designed based on the nominal plant, will make the transfer function from velocity command $u_{\nu}(s)$ to velocity response $\nu(s)$ become one. The block diagram can be simplified as Fig. 7. The system plant becomes $1 / s$ and has a $\mathrm{P}$ control in the position loop. Thus, increasing the $\mathrm{P}$ gain will make the system quickly move to the setpoint and decrease the steady-state error. The linear adaptive mechanism after the acceleration-deceleration period will be listed as

$$
\begin{cases}\rho_{\mathrm{i}}, & 0 \leq t<T_{\mathrm{acc}} \\ \rho_{\mathrm{i}}-\frac{\left(\rho_{\mathrm{i}}-\rho_{\mathrm{f}}\right) \cdot\left(t-T_{\mathrm{acc}}\right)}{\Delta T_{\text {tune }}}, & T_{\mathrm{acc}} \leq t \leq T_{\mathrm{acc}}+\Delta T_{\text {tune }} \\ \rho_{\mathrm{f}}, & t>T_{\text {acc }}+\Delta T_{\text {tune }}\end{cases}
$$

where $\rho_{\mathrm{i}}$ is the parameters before tuning, $\rho_{\mathrm{f}}$ denotes the parameters after tuning, $T_{\text {acc }}$ represents the acceleration-deceleration period in the positioning control, and $\Delta T_{\text {tune }}$ is the period of tuning parameters. The parameters in velocity loop and position loop controllers need to be tuned, and their values before and after tuning are described in the next section.

\section{Simulation And Experimental Results}

The experimental motion system illustrated in Fig. 8 consists of the following components: a linear-motor-driven motion system, a laser displacement meter, and a PC (PC1 in this figure) with a DAC and encoder interface. The linear motor system is composed of a linear motor (IL6-050A1) and an ac servo amplifier (SERVOSTAR CD) operating in torque (current) mode, both of which are made by Kollmorgen Corporation [14]. Two sensors are in use in this system, i.e., a linear scale (RENISHAW RGH24Y, resolution $1.0 \times 10^{-7} \mathrm{~m}$ ) which provides position information for the vector control of servo amplifier, and a fiber optic laser encoder (RENISHAW RLE10) for measuring the displacement of the motion table with adjustable resolutions. The accuracy of the resolution supplied by RLE10 is influenced by some environmental effects, such as relative humidity, temperature, pressure, and cosine errors. Therefore, calibration by another measurement instrument is required. The RENISHOW laser interferometer system, which includes an environmental compensation unit (EC10), is in use. After calibration, the basic length units for course and fine resolutions are found to be $7.91 \times 10^{-8}$ and $2.0 \times 10^{-8} \mathrm{~m}$, respectively. The choice of resolution scale is dependent on the encoder transition time ( $1 \mathrm{MHz}$ in our system), desired maximum velocity, and travel range. The sample rate is $2 \mathrm{kHz}$ $(T=0.0005 \mathrm{~s})$. The parameters of the motion system identified in our previous research [12] are listed in Table I. The original control parameters designed based on the cascade control are with $2-\mathrm{Hz}$ position controller bandwidth and $30-\mathrm{Hz}$ velocity controller bandwidth, and the original and the tuned parameters

$$
\begin{aligned}
X_{1}(s) & =\frac{1-e^{-T \cdot S}}{s} \cdot \frac{k_{\mathrm{a}} \cdot k_{\mathrm{t}}}{s \cdot(J \cdot s+B)} \cdot Y_{2}\left(z^{-1}\right) \\
X_{1}\left(z^{-1}\right) & =Z\left[\frac{k_{\mathrm{a}} \cdot k_{\mathrm{t}}}{s^{2} \cdot(J \cdot s+B)}\right] \cdot\left(1-z^{-1}\right) \cdot Y_{2}\left(z^{-1}\right) \\
X_{1}\left(z^{-1}\right) & =\frac{k_{\mathrm{t}} \cdot k_{\mathrm{a}} \cdot J}{B^{2}} \cdot \frac{\left(B \cdot T / J-1+e^{-B \cdot T / J}\right) \cdot z^{-1}+\left(1-e^{-B \cdot T / J}-B \cdot T / J \cdot e^{-B \cdot T / J}\right) \cdot z^{-2}}{1-\left(1+e^{-B \cdot T / J}\right) \cdot z^{-1}+e^{-B \cdot T / J} \cdot z^{-2}} \cdot Y_{2}\left(z^{-1}\right) \\
P\left(z^{-1}\right) & =\frac{k_{\mathrm{t}} \cdot k_{\mathrm{a}} \cdot J}{B^{2}} \cdot \frac{\left(B \cdot T / J-1+e^{-B \cdot T / J}\right) \cdot z^{-1}+\left(1-e^{-B \cdot T / J}-B \cdot T / J \cdot e^{-B \cdot T / J}\right) \cdot z^{-2}}{1-\left(1+e^{-B \cdot T / J}\right) \cdot z^{-1}+e^{-B \cdot T / J} \cdot z^{-2}}
\end{aligned}
$$




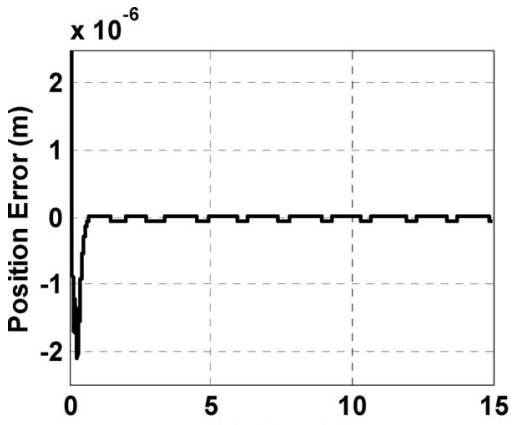

(a) time (s)

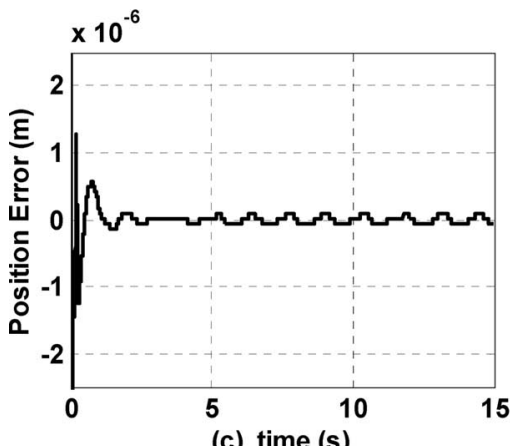

(c) time (s)

Fig 12. Position errors in Case (I) for the four controller structures. (a) TC. lower. (d) $\mathrm{TC}+\mathrm{FC}+\mathrm{DOB}$ with adaptive mechanism.

for removing limit cycles are listed in Table II. Moreover, for increasing the tracking performance, a linear adaptive mechanism is introduced, in particular (25). All the controlled parameters needed to be tuned are listed in Table III.

Simulation based on a motion control structure as shown in Fig. 9 with system parameters in Tables I-III is first executed to investigate the effect of each control components, i.e., traditional controller (TC), FC, and DOB in the steadystate positioning on the occurrence of limit cycles. Here, $Q_{1}$ for $2 \times 10^{-8}$-m quantizer of fiber optic laser encoder and $Q_{2}$ for 14-bit DAC quantizer are employed, and the S-curve position command is given as in (18) with $A=1 \times 10^{-3} \mathrm{~m}$ and $T_{\text {acc }}=0.25 \mathrm{~s}$. Six controller structures are performed, i.e., (a) $\mathrm{TC}$, (b) $\mathrm{TC}+\mathrm{DOB}$ with original parameters, (c) $\mathrm{TC}+$ DOB with adaptive mechanism, (d) $\mathrm{TC}+\mathrm{FC}$, (e) $\mathrm{TC}+\mathrm{FC}+$ $\mathrm{DOB}$ with original parameters, and (f) $\mathrm{TC}+\mathrm{FC}+\mathrm{DOB}$ with adaptive mechanism. Simulation results are shown in Fig. 10, where Fig. 10(a1)-(f1) are the position errors viewed in a large scale, and Fig. 10(a2)-(f2) are viewed in a small scale. Several observations are obtained in the following.

1) FC can improve only the tracking but not the final positioning.

2) Adding FC or not will not have any impact on the occurrence of limit cycles during state-state positioning.

3) The limit-cycle oscillation occurs in a DOB-based controller during state-state positioning, and this behavior can be eliminated when the adaptive tuned parameters in DOB controller are employed.

Next, to further verify the validity of our analytical results and the effectiveness of the adaptive mechanism, the experiments will be conducted for different controller structures and parameters.

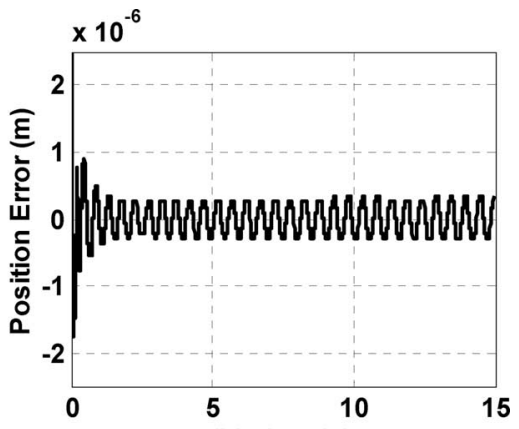

(b) time (s)

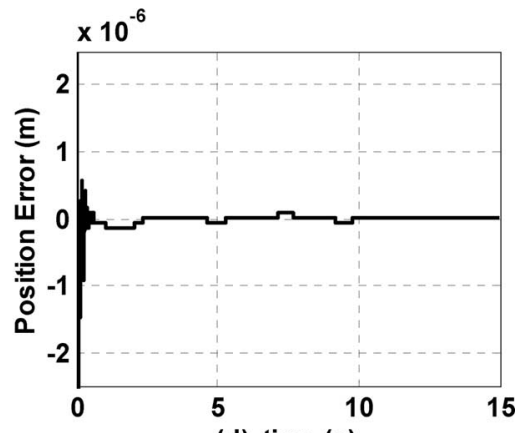

(d) time (s)
The S-curve position command in (18) is used. The indexes we use to evaluate the system performance are listed as follows:

$$
\begin{aligned}
& E_{\mathrm{tr}}=\sqrt{\frac{1}{N} \sum_{N}\left(x_{r}-x\right)^{2}}, \quad \text { for } 0 \leq t \leq t_{r} \\
& E_{\mathrm{qs}}=\sqrt{\frac{1}{N} \sum_{N}\left(x_{r}-x\right)^{2}}, \quad \text { for } t_{r}<t \leq t_{\mathrm{qs}} \\
& E_{\mathrm{ss}}=\sqrt{\frac{1}{N} \sum_{N}\left(x_{r}-x\right)^{2}}, \quad \text { for } t_{\mathrm{qs}}<t \leq t_{\mathrm{ss}}
\end{aligned}
$$

where $E_{\mathrm{tr}}$ is the rms index used to analyze the transient performance within the acceleration-deceleration period. $E_{\mathrm{qs}}$ is the rms index applied to analyze the system performance when converging to the final position. $E_{\mathrm{ss}}$ is the rms index for analyzing the steady-state performance and judge whether the controller can reduce or eliminate the limit cycles.

Case (I): As to the position command with $A=1 \times 10^{-3} \mathrm{~m}$ and $T_{\text {acc }}=0.25 \mathrm{~s}$, the time intervals for each index are $E_{\mathrm{tr}}$ for $0 \leq t \leq 0.25 \mathrm{~s}, E_{\mathrm{qs}}$ for $0.25 \leq t \leq 2.5 \mathrm{~s}, E_{\mathrm{ss}}$ for $2.5 \leq$ $t \leq 15 \mathrm{~s}$. The tuning period $\Delta T_{\text {tune }}$ in (25) is $0.5 \mathrm{~s}$. The encoder resolution we use in Case (I) positioning control is $2.0 \times 10^{-9} \mathrm{~m}$. Four controller structures, TC, TC $+\mathrm{FC}+\mathrm{DOB}$ with original parameters, $\mathrm{TC}+\mathrm{FC}+\mathrm{DOB}$ with only lower $B_{\mathrm{w}}(1 \mathrm{~Hz})$, and TC $+\mathrm{FC}+\mathrm{DOB}$ with adaptive mechanism are performed. Fig. 11 illustrates the position command, and the position errors resulted from the four types of controllers are shown in Fig. 12. From Table IV, the controller structure 4 with adaptive mechanism has the best overall performance. The transient performance of the controller structure 4 is as good as the controller structure 2 and 3 , and increases by 
TABLE IV

Performance IndeXes For the Four CONTROLler Structures in CASE (I)

\begin{tabular}{|l|c||c|c|}
\hline \multicolumn{1}{|c||}{ Controller structure } & $E_{t r}(\mathbf{m})$ & $E_{q s}(\mathbf{m})$ & $E_{s s}(\mathbf{m})$ \\
\hline \hline 1.TC(tradition controller) & $6.0971 \times 10^{-6}$ & $4.9790 \times 10^{-7}$ & $3.5595 \times 10^{-8}$ \\
\hline \hline 2.TC+FC+DOB(with original para.) & $1.4605 \times 10^{-6}$ & $3.5158 \times 10^{-7}$ & $2.3249 \times 10^{-7}$ \\
\hline \hline 3.TC+FC+DOB(only lower $B_{w}$ ) & $1.7968 \times 10^{-6}$ & $3.5505 \times 10^{-7}$ & $5.6946 \times 10^{-8}$ \\
\hline \hline 4.TC+FC+DOB(with adaptation) & $1.4448 \times 10^{-6}$ & $1.6204 \times 10^{-7}$ & $3.3081 \times 10^{-8}$ \\
\hline
\end{tabular}

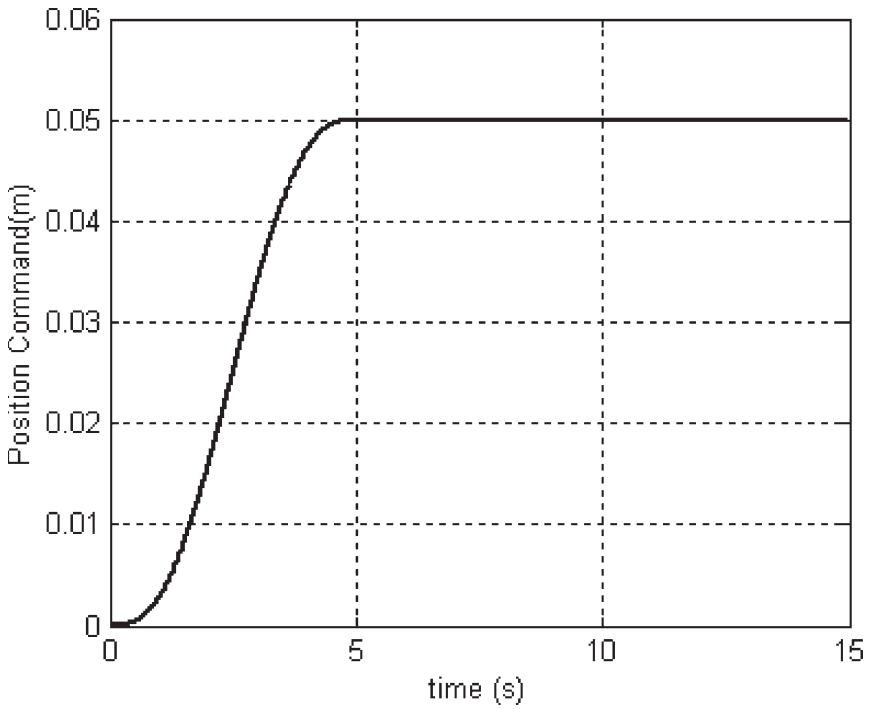

Fig. 13. Position command in Case (II).

$76 \%$ as compared with the controller structure 1 . Furthermore, the performance of converging to the final position for the controller structure 4 is almost two times better than the other three, and the steady-state error $\left(E_{\mathrm{ss}}\right)$ also decreases by $85 \%$ as compared with the controller structure 2 without the adaptive mechanism. As we can see from Fig. 12, the controller structure 3 with only lowered cutoff frequency $B_{\mathrm{w}}$ of the low-pass filter cannot avoid the limit cycles while reducing the amplitude and frequency of the limit cycles.

Case (II): For the position command with $A=5 \times 10^{-2} \mathrm{~m}$ and $T_{\mathrm{acc}}=5 \mathrm{~s}$, the time intervals for each index are $E_{\mathrm{tr}}$ for $0 \leq t \leq 5 \mathrm{~s}, E_{\mathrm{qs}}$ for $5 \leq t \leq 7.5 \mathrm{~s}$, and $E_{\mathrm{ss}}$ for $7.5 \leq t \leq 15 \mathrm{~s}$. The tuning period $\Delta T_{\text {tune }}$ in (25) is $0.5 \mathrm{~s}$. Three controller structures are performed, i.e., the $\mathrm{TC}, \mathrm{TC}+\mathrm{FC}+\mathrm{DOB}$ with original parameters, and $\mathrm{TC}+\mathrm{FC}+\mathrm{DOB}$ with adaptive mechanism. The $\mathrm{TC}$ is a controller structure that is identical to the one in Fig. 1 but without DOB and feed-forward-type FC. The encoder resolution we used in this case is $7.9 \times 10^{-8} \mathrm{~m}$. Fig. 13 illustrates the position command, and the position errors resulted from three types of controllers are shown in Fig. 14. From Table $\mathrm{V}$, we can see that the transient performance of the controller structure 2 increases by almost $57 \%$ as compared with the controller structure 1 (TC), but it has the worst performance in steady state because it generates the limit cycles as illustrated in Fig. 14.

The same as controller structure 2, the controller structure 3 has a good transient performance. Because controller structure 3 is with the adaptive mechanism, the performance of
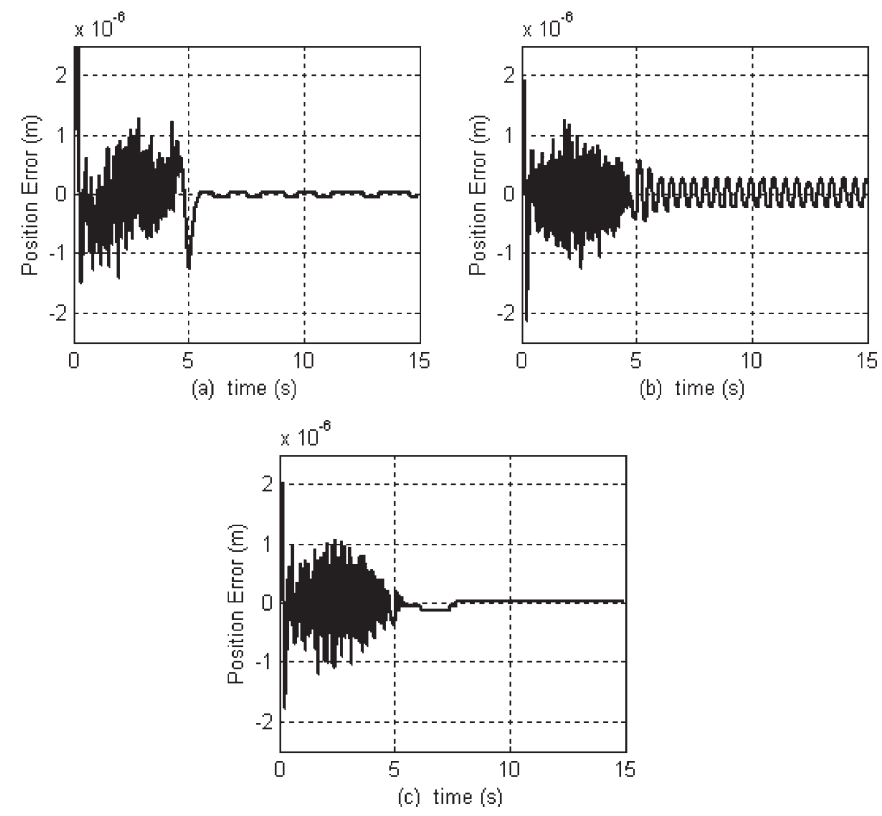

Fig. 14. Position errors in Case (II) for the three controller structures. (a) $\mathrm{TC}$. (b) $\mathrm{TC}+\mathrm{FC}+\mathrm{DOB}$ with original parameters. (c) $\mathrm{TC}+\mathrm{FC}+\mathrm{DOB}$ with adaptive mechanism.

converging to the final position $\left(E_{\mathrm{qs}}\right)$ and steady state $\left(E_{\mathrm{ss}}\right)$ is superior to the controller structure 2 and is the best of the three. As we can see in controller structure 2, the limit cycles caused by the mutual effect between the DOB and the quantization are the pure harmonic limit cycles (no dc components), so the assumption in Section II makes sense. The periodical behaviors of the controller structure 1 (TC) within 5-15 s are not identical with the limit cycles of the controller structure 2. Since the desired position $5 \times 10^{-2} \mathrm{~m}$ of the position command cannot be divided by the encoder resolution $7.91 \times 10^{-8} \mathrm{~m}$ with no remainder, there is always an offset error for the position. Under the circumstances, the effect of correcting the offset error continuously in the feedback loop causes a periodical response which has only one count drift at the final stage for controller structure 1. Controller structure 3 is not sensitive to the offset error because the gain of the velocity loop controller $C_{\nu}(s)$ has been reduced to a very low value.

\section{CONCLUSION}

In this paper, we introduce a sufficient condition of eliminating the limit cycles induced by the DOB and quantization. The sufficient condition enables us to design appropriate parameters in the velocity loop to avoid the limit cycles. However, we are 
TABLE V

Performance Indexes for the Three Controller Structures in CASE (II)

\begin{tabular}{|c||r|c||c|}
\hline \hline Controller structure & $E_{t r}(\mathbf{m})$ & $E_{q s}(\mathbf{m})$ & $E_{s s}(\mathbf{m})$ \\
\hline \hline 1.TC(traditional controller) & $1.0296 \times 10^{-6}$ & $2.9772 \times 10^{-7}$ & $4.0685 \times 10^{-8}$ \\
\hline 2.TC+FC+DOB(with original para.) & $4.3558 \times 10^{-7}$ & $2.5510 \times 10^{-7}$ & $1.8315 \times 10^{-7}$ \\
\hline \hline 3.TC+FC+DOB(with adaptation) & $4.2532 \times 10^{-7}$ & $1.1481 \times 10^{-7}$ & $2.3806 \times 10^{-8}$ \\
\hline
\end{tabular}

not satisfied about the performance of the parameters which conforms to the sufficient condition. We further introduce a linear adaptive mechanism that not only maintains the transient performance but also reduces the limit cycles in the steady state. As we can see from the experimental results in Section V, the $\mathrm{TC}+\mathrm{FC}+\mathrm{DOB}$ control structure with the adaptive mechanism has the best overall performance in positioning control.

\section{REFERENCES}

[1] K. Ohnishi, "A new servo method in mechatronics," Trans. Jpn. Soc. Electr. Eng., vol. 107, no. 4, pp. 83-86, 1987.

[2] T. Umeno and Y. Hori, "Robust speed control of DC servomotors using modern two degree-of-freedom controller design," IEEE Trans. Ind. Electron., vol. 38, no. 5, pp. 363-368, Oct. 1991.

[3] H. S. Lee and M. Tomizuka, "Robust motion controller design for highaccuracy positioning systems," IEEE Trans. Ind. Electron., vol. 43, no. 1, pp. 48-55, Feb. 1996.

[4] S. Komada, N. Machii, and T. Hori, "Control of redundant manipulators considering order of disturbance observer," IEEE Trans. Ind. Electron., vol. 47, no. 2, pp. 413-420, Apr. 2000.

[5] M. T. White, M. Tomizuka, and C. Smith, "Improved track following in magnetic disk drives using a disturbance observer," IEEE/ASME Trans. Mechatronics, vol. 5, no. 1, pp. 3-11, Mar. 2000.

[6] S. M. Shahruz, "Performance enhancement of a class of nonlinear systems by disturbance observers," IEEE/ASME Trans. Mechatronics, vol. 5, no. 3, pp. 319-323, Sep. 2000.

[7] M. Iwasaki, T. Shibata, and N. Matsui, "Disturbance-observer-based nonlinear friction compensation in table drive system," IEEE/ASME Trans. Mechatronics, vol. 4, no. 1, pp. 3-8, Mar. 1999.

[8] H. Kobayashi, S. Endo, S. Kobayashi, and C. J. Kempf, "Robust digital tracking controller design for high-speed positioning systems-A new design approach and implementation techniques," in Proc. IEEE Adv. Motion Control, 1996, vol. 1, pp. 65-70.

[9] M. Fischer and M. Tomizuka, "Application and comparison of alternative position sensors in high-accuracy control of an X-Y table," in Proc. IEEE Adv. Motion Control, 1996, vol. 2, pp. 494-499.

[10] T. Claasen, W. F. G. Mecklenbrauker, and J. B. H. Peek, "Frequency domain criteria for the absence of zero-input limit cycles in nonlinear discrete-time systems, with applications to digital filters," IEEE Trans. Circuits Syst., vol. CAS-22, no. 3, pp. 232-239, Mar. 1975.

[11] H. Olsson and K. J. Astrom, "Friction generated limit cycles," IEEE Trans. Control Syst. Technol., vol. 9, no. 4, pp. 629-636, Jul. 2001.

[12] Y. T. Shih, "The high precision control for a linear-motor-driven motion stage with friction compensation," Ph.D. dissertation, National ChiaoTung Univ., Hsinchu City, Taiwan, 2004.

[13] P. C. Edward, Digital Filtering. Boston, MA: Houghton Mifflin, 1992, ch. 9 .

[14] PLATINUM DDL and SERVOSTAR Setup Guide, Kollmorgen, a Danaher Motion Company, Radford, VA, Doc. M-LN-016-0702, 2002.

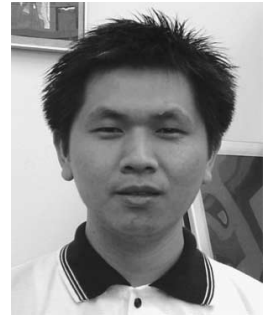

Rong-Hwang Horng (S'04) was born in Keelung, Taiwan, R.O.C., in 1973. He received the B.S. and M.S. degrees in mechanical engineering from National Chiao-Tung University, Hsinchu, Taiwan, in 1995 and 1997, respectively, where he is currently working toward the Ph.D. degree in mechanical engineering.

His research interests include modeling and control of CNC machine tool control technology.

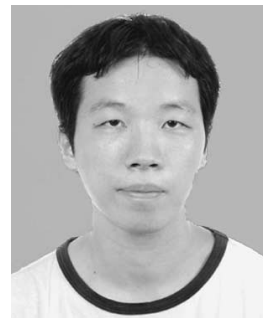

Heng-Lung Chou was born in Taipei, Taiwan, R.O.C., in 1979. He received the B.S. degree from National Taipei University of Technology, Taipei, in 2002, and the M.S. degree from National ChiaoTung University, Hsinchu, Taiwan, in 2004, both in mechanical engineering.

His research interests include modeling and control of CNC machine tool control technology.

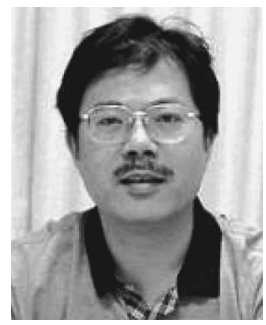

An-Chen Lee (M'04) was born in Chiayi, Taiwan, R.O.C., in 1956. He received the B.S. and M.S. degrees in power mechanical engineering from National Tsing-Hua University, Hsinchu, Taiwan, and the Ph.D. degree in mechanical engineering from University of Wisconsin, Madison, in 1986.

From 1986 to 1990, he was an Associate Professor with the Department of Mechanical Engineering, National Chiao-Tung University, Hsinchu, where he is currently a Professor. He also currently holds the Vice Dean and the Degree Program Chairman with the College of Engineering, National Chiao-Tung University. At National Chiao-Tung University, he teaches courses in automatic controls and digital controls. His current research interests are in $\mathrm{CNC}$ machine tool control technology, magnetic bearing technology, rotor dynamic and control, and production automation control.

Prof. Lee served as an Editorial Board member of the International Journal of Precision Engineering and Manufacturing, Chinese Society of Mechanical Engineers, and Journal of Applied Mechanics and Engineering. He is the recipient of the Chinese Society of Engineers Engineering Paper Award (1989), Excellent Research Award (1991-1992) of the National Science Council of Taiwan, Distinguished Research Award (1993-1994, 1995-1996, and 1997-1998) of the National Science Council of Taiwan, Distinguished Engineering Professor Award (1995) of the Chinese Society of Mechanical Engineers, and Distinguished Engineering Professor Award (2001) of the Chinese Institute of Engineers. He was a Research Fellow (1999-2004) of the National Science Council of Taiwan and holds the Chair Professor (2004) of National Chiao-Tung University. 